\title{
Smart home-driven digital memory notebook support of activity self-management for older adults
}

\author{
Jessamyn Dahmen BS ${ }^{\mathrm{a}, *}$ \\ Bryan Minor $\mathrm{PhD}^{\mathrm{a}}$ \\ Diane Cook $\mathrm{PhD}^{\mathrm{a}}$ \\ Thao Vo BS ${ }^{b}$ \\ Maureen Schmitter-Edgecombe $\mathrm{PhD}^{b}$
}

\begin{abstract}
aWashington State University, School of Electrical Engineering and Computer Science, Pullman, WA, USA; ${ }^{b}$ Washington State University, Department of Psychology, Pullman, WA, USA; *Corresponding author: jb3dahmen@wsu.edu
\end{abstract}

\begin{abstract}
J. Dahmen, B. Minor, D. Cook, T. Vo, M.S. Edgecombe. Smart home-driven digital memory notebook support of activity self-management for older adults. Gerontechonology 2018;17(2):113-125 https://doi.org/10.4017/gt.2018.17.2.005.00 Memory notebooks represent compensatory strategies that can be used to help individuals with memory impairments improve everyday functioning and compensate for problems created by lapses in memory. These external memory aids have been shown to help individuals record daily events in a structured manner, support retrospective and prospective memory, and reduce cognitive load. Although digital memory aids abound, they require a level of programming on the part of the user that is not practical for individuals with cognitive impairment. In this paper, we introduce a digital memory notebook (DMN) app that partners with a smart home to inform individuals about the activities they already performed and to notify them at appropriate times about the activities they still need to complete. We perform several iterative usability studies evaluating the DMN app interface and pilot test the smart home features in a live smart home testbed. Our iterative design process found that the components of the DMN app were generally usable by older adults and that satisfaction with the interface improved based on iterative feedback. Based on these results we anticipate that the proposed DMN app and smart-home partnership will be an effective technology for helping older adults with memory impairment improve everyday functioning.
\end{abstract}

Keywords: smart environments, human computer interfaces, digital memory notebook

\section{INTRODUCTION}

By 2050 it is estimated that 1.5 billion people ( $16 \%$ of the world's population) will be age $65+1$. A large proportion of this older adult population will experience cognitive decline $e^{2,3}$. Older adults with mild cognitive impairment $(\mathrm{MCl})$ and other neurologic disorders often need assistance remembering to complete everyday activities, such as taking medication or getting to an appointment, as a result of cognitive difficulties. With such a large proportion of the population needing assistance, health care costs have risen and resources to care for these older adult individuals have become more limited ${ }^{4}$. This situation coupled with the fact that older adults want to stay in their own homes punctuates the need for advancements in smart home-related technologies that can be used as cost effective and efficient tools to sup- port older adults with cognitive impairment, their families, and their care providers.

As a frequent symptom of cognitive impairment, memory deficits can significantly reduce functional ability in everyday situations leading to loss of independence and decreased quality of life ${ }^{5}$. A variety of clinically validated compensatory strategies have been developed to help individuals with memory impairments. Among these strategies, pen-and-paper memory notebooks have successfully been used in cognitive rehabilitation and can effectively be incorporated into daily routines ${ }^{6,7}$. As smart home and activity recognition technologies mature, the potential for augmenting traditional paper-based memory notebooks with technology is apparent. Digital interfaces can increase the availability and rich- 
ness of data captured in natural home environments ${ }^{8,9}$. Furthermore, activity recognition techniques can be used as a "cognitive prosthesis" to support functional independence.

Recent advances have made smart home technologies a reality. A smart home (or another smart environment) is defined as one that is able to acquire and apply knowledge about the physical environment and its inhabitants in order to improve their experience in that environment ${ }^{10,11}$. An important piece of information for a smart home to acquire is the activities that residents perform in the home because activities provide a rich vocabulary to express human behavior ${ }^{8,9}$. Correspondingly, automated activity recognition and prediction allow smart environments to detect and forecast activities (e.g., medications, grooming) in real time ${ }^{12-14}$. However, much of the current research does not fully utilize this information to assist residents and support self-management of daily routines in meaningful ways.

In this paper, we introduce a digital memory notebook (DMN) mobile application that utilizes smart home data and activity recognition techniques to automatically help users complete daily tasks. The goal of this paper is to introduce a real-time automated intervention that partners mobile apps with smart home-based activity learning using two primary mechanisms. First, the DMN automatically checks off completed tasks using smart home data to reassure individuals of tasks they have completed. Second, the DMN also predicts when tasks should be completed and utilizes this information to prompt users to initiate activities at the times they normally occur, without user programming.

The DMN's ability to incorporate smart home data to support every day task completion establishes a unique partnership with activity recognition systems used in smart homes. This partnership can be used for effective interventions that can help remind individuals to use the DMN and initiate important activities. These smart home powered features automatically help reduce memory load and help aid in self-management and self-efficacy by prompting for the completion of critical activities of daily living such as eating and medication adherence. We detail our iterative design process for creation of the DMN user interface with older adult participants and summarize results of testing the technology in an actual smart home.

\section{SCEnARIOS}

We motivate the design of a DMN through a series of scenarios that illustrate how the DMN supports older adults with memory impairments in home settings. The features of the memory notebook are briefly described here - the design and implementa- tion of these features are described in Methods.

\section{Scenario 1: General use}

Mary is a 66-year-old woman who is starting to experience mild memory difficulties. Mary frequently forgets to write down important events such as her grandson's birthday. Mary has used other task scheduling apps in the past but has found them overwhelming to use and difficult to see. Recently, Mary has discovered the DMN app which utilizes large print and contrasting colors. The DMN app provides familiar scheduling interfaces such as a to-do list and calendar and is designed to be accessible to those who are not very familiar with mobile technology. Mary can schedule her events using a simple interface and visualize when events are due using time blocks and marked calendar days. Mary can also use the DMN to record important medical information such as her medication regimen and doctor's contact information as well as to journal about unusual or important events occurring that day that she wants to remember.

\section{Scenario 2: Activity recognition}

Bob is a 70-year-old man who has recently been diagnosed with mild dementia. Bob has several motion sensors installed in his apartment and recently installed the DMN app on his tablet. As a result of his memory limitations, Bob frequently has trouble initiating eating meals and recalling whether he has already eaten. One of his favorite features is the DMN app's ability to communicate with the smart home to tell him when the smart home has detected activities as he performs his daily routine. The DMN's smart homepowered back-end then adds the smart home detected activities and their detection time to his to-do list. Whenever Bob is not completely sure if he has eaten, he now refers to the DMN app to help jog his memory and to assure him of the activities that he already performed that day. This allows him to perform important activities with more independence and confidence.

\section{Scenario 3: Activity prediction}

Jenny is a 71-year-old woman who has been experiencing memory difficulties. She often cannot remember to take all her medication on time. This has significantly impacted her daily functioning, as many of her medications need to be taken at specific times throughout the day to avoid side effects. Jenny also has several motion sensors installed in her home. She has been taking medicine regularly during dinner when her husband reminds her, but recently downloaded the DMN app onto her tablet and wants to do this task more independently. She enjoys using the DMN app as it can automatically predict when important activities should be completed based on her past (husband-assisted) behavior. The smart home- 
assisted DMN app predicts when she takes her medicine. It also alerts her when the time to complete the activity is getting closer by using a color highlight on the day's to-do list and if she forgets the activity (e.g., she completes dinner without taking her medicine), the DMN app prompts her with an audio alert and reminder.

\section{Related Work}

There has been a variety of work on paper-based memory notebooks and digital task scheduling memory aids. Traditional memory notebooks are external memory aids that have been shown in clinical studies to be effective compensatory strategies for older adults with memory impairments. The term "memory notebook" usually refers to a diary or scheduling system where individuals log aspects of their daily lives and activities. For example, a memory notebook designed by Schmitter-Edgecombe et al. consisted of a daily log where participants could schedule and record activities on an hourly basis, a to-do list, calendar, personal notes, current work, and goals ${ }^{6}$.

The goal of traditional memory notebooks is to support everyday functioning by providing external information that can be used to compensate for lapses in memory. For example, earlier studies assessed memory notebooks as a cognitive intervention for individuals with Traumatic Brain Injury (TBI) and individuals with mild dementia $^{6,15}$. Currently, for individuals with TBI and stroke, recommended training in the use of external compensatory devices, such as a memory notebook, is a Practice Standard ${ }^{16}$. In one randomized controlled trial for 46 individuals with $\mathrm{MCl}$ and their care-partners, individuals who used the notebook intervention reported fewer symptoms of depression and greater use of memory strategies in their everyday lives, while carepartners reported better coping self-efficacy ${ }^{17}$.

Research related to digital versions of traditional clinically validated memory notebook systems is limited. However, several studies explore how digital memory aids can be used to support memory in a broader context. For example, digital memory aids have been used to augment memory for populations that do not necessarily have significant memory impairments ${ }^{18}$. This study used a wearable "memory prosthesis" that recorded audio and contextual information from conversations and provided computer-based and wearable-based retrieval tools to help users access memories.

Furthermore, these aids have also been applied to creating systems such as "personal digital memory stores" that organize multi-media extracted from people's lives. These systems can be used for more social, therapeutic and personal purposes to improve quality of life rather than specifically focusing on improving everyday functioning. Tan et. al. described how a large personal store of digital information could be used to support memory ${ }^{19}$. Czerwinski et. al. explored how users might use and store a life's worth of digital memories produced by digital memory technologies such as wearable cameras and biometric sensors ${ }^{20}$. The majority of these studies find that digital memory aids are effective ways to augment memory in everyday situations.

Tsui and Yanco describe digital approaches used for task sequencing such as digital picture books or task prompters ${ }^{21}$. Kapur et al. also review digital memory aids and examine electronic organizers and reminder systems. While these systems are not specifically based on clinically validated memory notebooks, the overlaps in functionality are clear ${ }^{22}$. However, these aids are still driven primarily by the user and do not automate information based on smart home data or detected activities. While many aspects of these digital aids are still in development, primary goals for digital memory aid system development include providing (a) automatic recording of memories, (b) storage of memories in large personal memory stores, and (c) convenient access to the memories of interest.

In earlier work, Feuz et al. investigated how machine learning techniques can be used to remind individuals to reference and use an external aid such as a digital memory notebook ${ }^{23}$. Rather than focusing exclusively on simple time-based prompts, in this study prompts were activated during activity transition points. This study compared the activity transition-based prompts to the time-based prompts to see if the activity-based prompts would increase responsiveness and perceived helpfulness. Other studies have also used similar approaches to prompting. One study used a smart phone based system combined with machine learning based "break-point" detection to decide when to provide prompts ${ }^{24}$. Other studies focused on using context aware techniques combined with wearable technologies to detect transitions between activities ${ }^{25}$. Overall, these studies found that transition-based prompting systems can improve the efficacy of the memory and task completion aids they were partnered with by not interrupting users while they are performing tasks, thus lessening the cognitive load.

In this paper, we discuss how smart home data can be used to augment the functionality and interface of a DMN app intervention. This work is unique in that it partners smart home-based activity recognition and mobile app design to create an activity-aware memory intervention. This is the first intervention of which we are aware that integrates smart home-sensed information into a mobile intervention tool. It is 


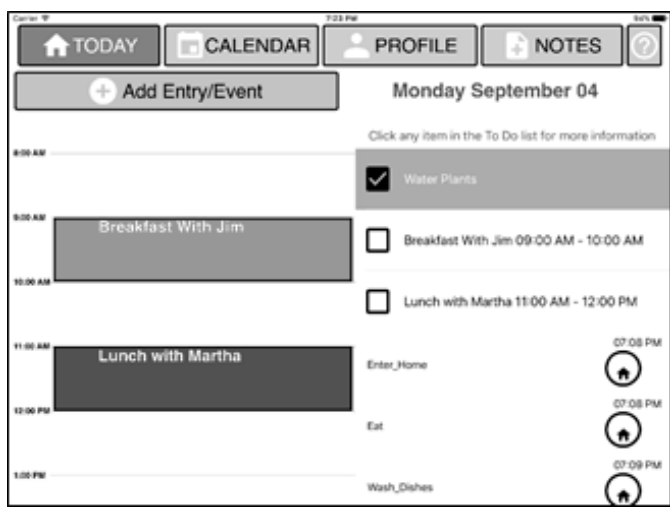

Figure 1. The Home Page interface. The Home Page's button is labeled as "Today" when showing the current date

also one of the few instances in which activity recognition is not only implemented and evaluated but is used as a component in a larger intervention strategy.

\section{Methods}

Our methods have two main components. First, we design a notebook interface that will make it appealing and usable by older adults experiencing memory difficulties. Second, we partner the memory notebook with a smart home in order to provide activity-aware information in the moment that can improve functional independence for older adults and those experiencing memory limitations.

Our DMN app was designed using an iterative feedback process with older adults and caregiver participants. The DMN is an application designed to be used on a capacitive touchscreen tablet. The results of these iterative usability studies are summarized in Results. The design of each aspect of the DMN app was driven by participant feedback and guidance from the literature. We use criteria established by Díaz-Bossini and Moreno in creating mobile applications for older adults to guide the design of the $\mathrm{DMN}^{26}$.

\begin{tabular}{|c|c|c|c|c|c|c|c|}
\hline TODAY & & DCALENDAR & \multicolumn{2}{|c|}{ PROFILE } & \multicolumn{2}{|c|}{ + NOTES } & 10 \\
\hline \multicolumn{7}{|l|}{$<$} & $>$ \\
\hline \multicolumn{8}{|c|}{ September 2017} \\
\hline Sun & Mon & Tue & Wed & Thu & $\mathrm{Fri}$ & $\mathrm{Sa}$ & \\
\hline 27 & 28 & 29 & 30 & 31 & 1 & 2 & \\
\hline 3 & (4) & 5 & 6 & 7 & 8 & 9 & \\
\hline 10 & 11 & 12 & 13 & 14 & 15 & 16 & \\
\hline 17 & 18 & 19 & 20 & 21 & 22 & 23 & \\
\hline 24 & 25 & 26 & 27 & 28 & 29 & $3 c$ & \\
\hline 1 & 2 & 3 & 4 & 5 & 6 & z & \\
\hline \multicolumn{8}{|c|}{ Go To Today } \\
\hline
\end{tabular}

Figure 2. The Calendar Page interface
In this section, we briefly describe the major components of the DMN app and also highlight how design criteria for older adults were employed when designing the DMN app. We also extend a mobile intervention to a more comprehensive intervention system by integrating smart home capabilities with the mobile interface.

The DMN is composed of four main components: Home, Calendar, Profile, and Notes. These components were chosen based on a clinically-tested version of a paper-based memory notebook ${ }^{15}$. One of the main features of the DMN contained within the Home component is the automatic incorporation of smart home information into the DMN's interface to automatically detect tasks and predict when tasks should be completed. These features help the DMN serve as a real-time automated intervention that can act on smart environment data in supportive and meaningful ways.

\section{Description of main components}

The first of the four main DMN visual components is the Home, which is divided into two main sections. The to-do list on the right includes userspecified tasks as well as smart home-detected and predicted tasks (explained in more detail in Activity Recognition and Activity Prediction). The left pane of the home page interface is referred to as the hourly view, which shows visual blocks of time associated with a task.

Figure 1 shows a screenshot of the Home component. The DMN uses large selectable interface items such as buttons and checkboxes in the Home as well as throughout the rest of the app's interface. As recommended for older adults, these items provide large targets to help compensate for mobility or visibility related issues ${ }^{26}$. There is also clear confirmation of target selection as each item clearly opens a new view. Only single tap, swipe and scroll gestures are used for interaction. Unlike many commercial generaluse apps, color is used sparingly in the DMN to highlight important components and contrasting colors are used. All DMN icons are simple to help clarify function.

The Calendar component of the DMN allows users to easily navigate to different dates on the home page by selecting them. An orange dot is used to indicate whether a specific date has scheduled tasks on it. Figure 2 shows the Calendar. The selection spaces for each date provide large targets for users to tap. Color is used sparingly to highlight important items such as the current date.

The Notes component of the DMN allows users to type notes associated with each day of the calendar year. Users can keep notes about their thoughts throughout the day. The Notes feature 


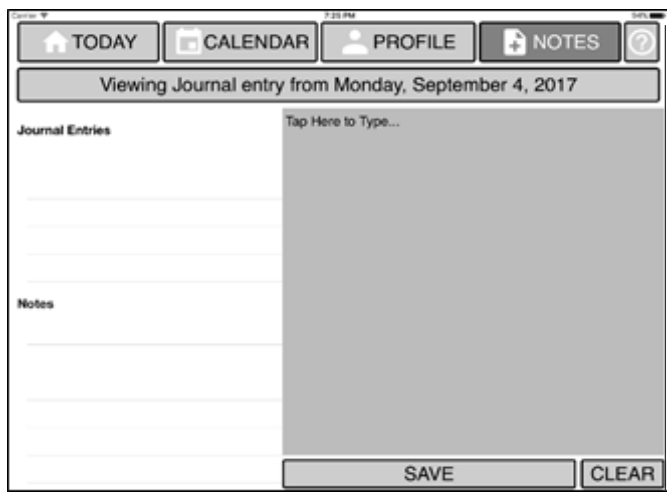

Figure 3. The Notes Page interface

supports the journaling functionality that was explored in paper versions of the memory notebook $^{15}$. Figure 3 shows a screenshot of the Notes page. This component uses simple language and large targets to help users easily take notes throughout their day.

The Profile component allows users to enter personal information such as their doctor's contact and personal medical information. This component helps users remember this critical information as well as informing care providers. Figure 4 shows the Profile page. This page uses a simple layout and large text field targets.

\section{Activity recognition and prediction system overview}

In order to perform real-time activity recognition and prediction, the DMN app relies on a Pythonbased back-end that partners with the existing smart home infrastructure. Figure 5 shows a diagram of the components that are used to drive the smart home functionality for the DMN app.

In this infrastructure, a RabbitMQ (https://www.rabbitmq.com) message broker sends a stream of messages in real time from the smart home to the DMN containing automatically-recognized activity labels

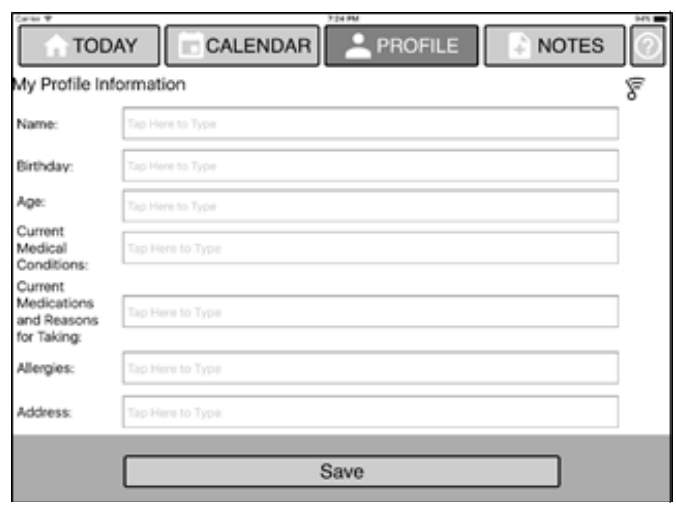

Figure 4. The Profile Page interface and automatically-generated predictions as residents perform their daily routines. When a sensor event is triggered (e.g., when a person moves under a motion sensor), the sensor event information is sent as input to the activity recognition and activity prediction algorithms described in Activity Recognition and Activity Prediction. These algorithms use the sensor data to provide a new recognized activity label and an updated set of predicted activity times. These activity labels and prediction values are sent to a Python script that represents an event listener and are stored in a database. To receive the latest recognized activities and predictions the DMN app sends requests to a Flask web application (http://flask.pocoo.org) implemented using a Python script. This component gathers smart home information such as the activity labels, timestamps, and predicted times for each activity from the application database. The Flask application also stores information about user interaction with the DMN app, such as the user confirming that they have completed a smart home task and the timestamp when they confirmed it.

\section{Smart home partnership: Activity recognition}

The smart home provides valuable information for self-management of daily routines through sensor data collection and automatic recognition of activities. Building on this partnership, the DMN automatically incorporates routine activities and to-do list tasks that can be recognized as completed by our smart home. Figure 6 shows screenshots of the application's to-do list populated by recognized smart home activities. These "smart home tasks" are distinguished by a distinct layout compared to regular user-entered to-do list tasks and are marked by a home icon to help users identify smart home recognized tasks. By pressing the home icon the user can confirm that the smart home correctly identified a completed task. When an activity is recognized the DMN also provides an audio tone. A demonstration of this capability is available featuring a previous iteration of the interface (Video available at: https://www.youtube.com/ watch? $v=$ mO7GCqgGBGk).

The purpose of this feature is to help individuals who experience memory impairments keep better track of activities they are completing throughout the day and thus manage their daily routines more independently. This feature utilizes the unique partnership between the DMN and the smart home activity recognition capabilities. Indicating that the smart home has recognized a task as completed reduces cognitive load and maintains everyday functional independence by helping users recognize that they have completed tasks rather than having to rely on their own memory or ability to consistently schedule activities. In this way, the DMN helps adults with cogni- 
Table 1. Activity recognition and activity prediction features; * are used for activity prediction only.

\begin{tabular}{ll}
\hline Feature & Description \\
\hline lastSensorEventHours & Hour of day \\
\hline lastSensorEventSeconds & Seconds since midnight \\
\hline windowDuration & Length of window (seconds) \\
\hline timeSinceLastSensorEvent & Seconds since previous event \\
\hline prevDominantSensor1 & Most frequent sensor in previous window \\
\hline prevDominantSensor2 & Most frequent sensor two windows ago \\
\hline lastSensorlD & Current event sensor \\
\hline lastLocation & Most recent sensor location \\
\hline sensorCount & Number of events in window for each sensor \\
\hline sensorElTime & Elapsed time since each sensor fired \\
\hline laggedTimestamp* & Previous event time stamps \\
\hline laggedPredictions* & Previous event predictions \\
\hline
\end{tabular}

7 shows the placement of sensors at a CASAS smart home test bed called Kyoto. The activity recognition feature was tested using data generated from this residence. These sensors provide a sequence of time-stamped sensor events while the smart home resident performs her daily routines. Both the activity recognition and activity prediction features are capable of incorporating live smart home test bed sensor data.

tive impairment to improve functional independence by supporting retrospective memory.

In order to automatically notify users of recognized tasks, the DMN app needs to receive information about activities that are recognized by a smart home outfitted with ambient sensors. The activity recognition algorithm we use, called $A R$, is described in the literature ${ }^{27,28}$. The AR algorithm can utilize information from many different types of ambient sensors to model and recognize activities in real time. For our implementation of the $\mathrm{DMN}$ and its partnership with a smart home, the smart home contains sensors placed around the home including motion sensors and door sensors. These sensors report changes in their binary states such as On/Off and Open/Closed. Figure

Our activity recognition algorithm, AR, automatically provides activity labels for these sensor events. These activities represent activities of daily living such as cooking, eating, and sleeping. The algorithm identifies activity labels in real time using a set of predefined activities. Initially, human annotators label one month of sensor data from each smart home with predefined activity labels to provide the ground truth activity labels that can be used to train the activity recognition model. This trained model is then used to provide the activity labels for the smart home sensor events.

AR maps a sequence of sensor events onto an activity label ai, where the label is drawn from a predefined set of $K$ activities $A=\{A 1, A 2, \ldots$,

Table 2. Activity recognition confusion matrix for activities: bed-toilet transition (BT), sleep (SL), personal hygiene ( $P H)$, bathe (BA), take medicine $(T M)$, cook (CO), eat $(E A)$, wash dishes $(W D)$, relax $(R E)$, leave home $(L H)$, enter home $(E H)$, and work (WO)

\begin{tabular}{lllllllllllll}
\hline & BT & SL & PH & BA & TM & CO & EA & WD & RE & LH & EH & WO \\
BT & 3224 & 1 & 4 & 0 & 0 & 0 & 0 & 0 & 0 & 0 & 0 & 0 \\
\hline SL & 1 & 5536 & 0 & 0 & 0 & 0 & 0 & 0 & 89 & 0 & 0 & 2 \\
\hline PH & 15 & 1 & 19533 & 2 & 78 & 0 & 0 & 0 & 0 & 2 & 0 & 0 \\
\hline BA & 0 & 0 & 13 & 2377 & 0 & 0 & 0 & 0 & 0 & 0 & 0 & 0 \\
\hline TM & 0 & 0 & 40 & 0 & 1232 & 12 & 0 & 2 & 0 & 0 & 0 & 0 \\
\hline CO & 0 & 0 & 0 & 0 & 90 & 29545 & 19 & 32 & 0 & 0 & 0 & 0 \\
\hline EA & 0 & 0 & 0 & 0 & 0 & 1 & 1792 & 0 & 0 & 0 & 0 & 6 \\
\hline WD & 0 & 0 & 0 & 0 & 1 & 22 & 17 & 5214 & 0 & 0 & 0 & 2 \\
\hline RE & 0 & 0 & 0 & 0 & 0 & 0 & 0 & 0 & 2652 & 0 & 2 & 23 \\
\hline LH & 0 & 0 & 0 & 0 & 0 & 0 & 0 & 0 & 0 & 895 & 0 & 0 \\
\hline EH & 0 & 0 & 0 & 0 & 0 & 0 & 0 & 0 & 0 & 0 & 948 & 0 \\
\hline WO & 0 & 0 & 0 & 0 & 0 & 4 & 33 & 0 & 0 & 0 & 0 & 671 \\
\hline
\end{tabular}

$A K\}$ and ai corresponds to the ith activity class. AR generates a label that indicates the activity that was performed when the last sensor event was generated. To generate an activity label for smart home sensor events, AR uses a windowing approach. Let s1, s2, . . . , $\mathrm{sN}$ to represent the sequence of sensor events. The sequence $\mathrm{s} 1, \mathrm{~s} 2, . ., \mathrm{sN}$ is divided into windows. A window Wj can then be 


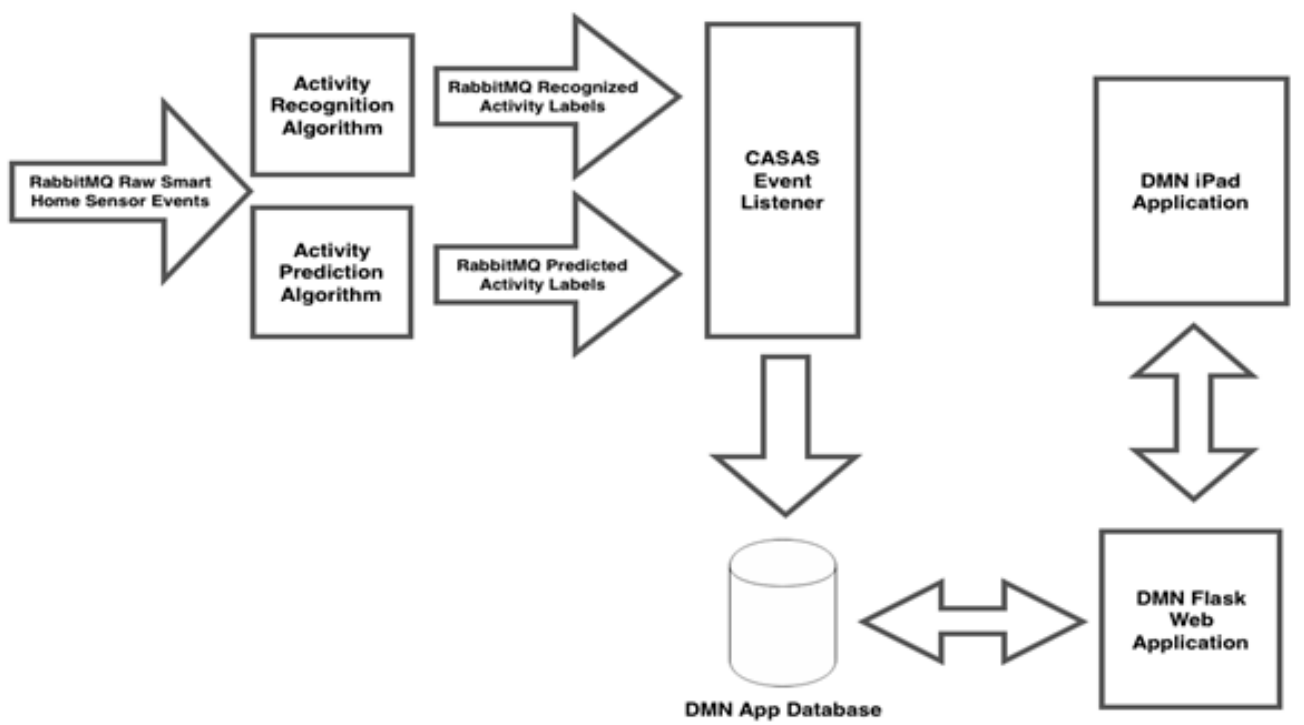

Figure 5. A high level system component overview of the DMN live smart home features

represented by the sequence $[\mathrm{sj}, \mathrm{sj}+\Delta \mathrm{s}]$ where $\Delta \mathrm{s}$ varies depending on experimental context. Once $W_{j}$ is defined, features "x belongs to $R^{d}$ " are extracted from the corresponding sensor data input. This feature vector $x j$ captures the time of the first and last sensor events, the temporal span of the window Wi and a count of the different sensor events within the window. The full set of features that are extracted for activity recognition and for activity prediction is shown in Table 1. Each xj is mapped onto a label yj, which corresponds to an activity class in A and designates the activity that occurred at the end of the sensor event sequence. The $x \mathrm{j}$ and the corresponding yj then become the training data that is given to a classifier for activity model learning.

In a previous study, the accuracy of AR was measured for a collection of data from smart home-based activities and yielded 98\% accuracy using 3 -fold cross validation ${ }^{28}$. The evaluation of DMN in this paper focuses on one home with a single resident. To demonstrate the performance of AR activity recognition for a single-resident home in which a DMN might be used, we

Work

Figure 6. DMN screenshot shows a list of detected activities derived from live smart home test bed sensor data tested it in a smart home testbed for recognition of 12 activities based on 2 months of data. The data was collected continuously while the resident performed normal daily routines. External annotators used the floorplan, sensor data, and resident-completed surveys of typical routines to label the data with ground truth activity labels. Activity recognition accuracy for 12 activities was $99.3 \%$ using 3 -fold cross validation. The confusion matrix for this experiment is shown in Table 2. In addition to validating activity recognition based on historic data, we also validate the algorithm using verification from smart home residents themselves "in the moment", using ecological momentary assessment (EMA). The EMA app occasionally queries the resident about their current activity and we use the response as ground truth to measure the performance of AR. Based on this validation mechanism for 8 users for 11 activities, we observed a $86 \%$ accuracy $^{29}$.

\section{Activity prediction}

The DMN app also partners with the smart home to predict when activities or tasks should be completed. The purpose of this feature is to help older adults with memory impairment remember to initiate activities and prompt them with a visual reminder indicating the activity is close to its predicted initiation time. Prompting thus helps to promote everyday functional independence for older adults by improving prospective memory. This feature utilizes the DMN smart home partnership by automatically visually prompting users to use the DMN to help initiate important tasks such as taking medication on time. Figure 8 shows the interface for the predicted tasks. As the time gets closer to the predicted time for the 


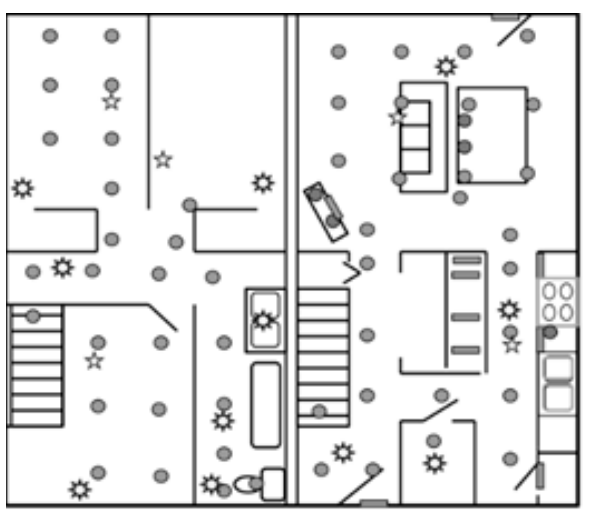

Figure 7. The layout of the CASAS smart home test bed that was used to test smart home features.

Sensor Key: task to be completed, the border changes to red indicating that the time the task is "due" is getting closer. This helps support everyday functioning, by visually prompting the user to complete important tasks. The current version of the DMN incorporates smart home events using predicted activity times derived from real smart home user data and an activity prediction algorithm.

Here we briefly describe the algorithm we created to generate predicted activity timestamps, additional detail is found in the literature ${ }^{30}$. In activity prediction, we consider the problem of predicting future activity occurrence times from sensor data. As with activity recognition, A refers to the set of activities. Given features " $x$ belongs to $\mathrm{R}^{\mathrm{d}^{\prime \prime}}$ extracted from sensor data input, an activity predictor generates $\hat{z}=\left(\hat{z}_{1}, \hat{z}_{2}, \ldots \hat{z}_{k}\right)$ as output, where $\hat{z}_{i} \in \Re^{d}$ is the predicted relative occurrence time for activity $a_{j}$. That is, $z_{j}$ is the predicted number of time units that will elapse from the current sensor event until $a_{j}$ next occurs. The features $x$ used as input to the predictor are generated from a recent window of observed sensor events. Unlike commercially-available reminder apps, the activity predictor can prompt individuals based on the context in which they normally perform an activity. For example, if a resident takes medicine with dinner, the home can recognize that they are eating and prompt them to take medicine at that time.

A basic activity predictor is the Independent Predictor (IP). This predictor uses a separate regression model for each activity to be predicted. (Any multivariate regression model can be used, although in our implementation we utilize random forest regressors because of their superior performance on this data.) Given a set of labeled sensor events, we create input-output training pairs $\left\{\mathrm{x}_{\mathrm{i}}, \mathrm{z}_{\mathrm{ij}}{ }^{*}\right\}$ which consist of the feature vector and corresponding ground-truth time for activity aj. The full set of activity prediction features is shown in Table 1.

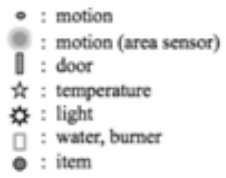

These training examples are then used to train the regression learners separately. The trained regressors then continuously predict the times for all activities. IP can be enhanced by using previous activity predictions to inform future predictions creating a Recurrent Activity Predictor (RAP). Whereas the features used for IP consist only of local features $x_{\text {local }}$ drawn from the sensor events, RAP also utilizes additional context features $x_{\text {context }}$ drawn from previous activity predictions. For activity $a_{j}$, the context features consist of previous predictions $\mathrm{z}$ from $\mathrm{H}$ previous events for all activities except $a_{j}$. Since events are not spaced equally in time, the elapsed time since the previous event is also included in the context features. The inclusion of the activity context allows RAP to learn arbitrary relationships between activities. RAP is trained and utilized in a similar manner to IP. RAP yielded a mean absolute prediction error of less than one hour for data collected from $(\mathrm{N}=30)$ testbed homes ${ }^{30}$.

The activity forecasting algorithm was validated in the context of activity prompting for nine homes with a number of activities ranging from 2 to $9^{29}$. In this evaluation, we observed a rangenormalized mean absolute error of 0.02 seconds.

\section{Results \\ Usability testing results}

The DMN interface has undergone three iterations of usability testing sessions. Each session included 4-8 participants with a total of $\mathrm{N}=17$ participants. The DMN interface presented in this paper is based on Iteration 3. Participants included caregivers, cognitively healthy older adults, and older adults with $\mathrm{MCl}$. Table 3 shows a summary of participant demographics for each iteration.

Between each iteration, the DMN was modified based on user feedback and new features were added. Iteration 1 included the basic Home, Profile, and Calendar components of the system. Iteration 2 also included the Notes component as well as questions about the automatically detected smart home tasks. Iteration 3 included questions about activity prediction. Based on feedback from Iteration 1, in Iteration 2 buttons and text were made bigger throughout the app for better visibility. Iteration 3 included questions about activity prediction and further improvements to text and interface visibility. All testing sessions were conducted with a trained administrator. 
Table 3. Participant demographics by iteration

\begin{tabular}{llll}
\hline & Iteration 1 & Iteration 2 & Iteration 3 \\
\hline Number of Participants & $\mathrm{N}=8$ & $\mathrm{~N}=4$ & $\mathrm{~N}=5$ \\
\hline Mean Age in years & 71.25 & 72.25 & 71.40 \\
\hline Age Range in years & $46-94$ & $62-94$ & $67-77$ \\
\hline Description & 1 Caregiver, & $2 \mathrm{TBI}, 1 \mathrm{MCl}$, & 2 Caregivers, \\
& 7 Healthy older adults & 1 Caregiver & $3 \mathrm{MCl}$ \\
\hline Gender & 5 female, & 3 female, & 4 female, \\
& 3 male & 1 male & 1 male \\
\hline $\begin{array}{l}\text { Education range in } \\
\text { number of years }\end{array}$ & $13-20$ & All 20 & $14-18$ \\
\hline
\end{tabular}

participants that gave that response and then summing these values.

The sum was then divided by the number of participants in that iteration to account for unequal sample sizes. The aggregate scores for Iterations 1, 2, and 3 were $9.5,11.5$, and 8.6 , respectively. From Iteration 1 to 2 there was a percent increase of $+10.53 \%$. From Iteration 2 to 3 there was a percent decrease of $-25.22 \%$. From Iteration 1 to 3 there was a percent decrease of $-9.47 \%$. Percent decreases are preferable.

Table 4 shows how participants responded when asked to rate the confirm smart home task scenarios in terms of ease of task completion. In this scenario, participants were asked to imagine the app was paired with smart home technology that could automatically recognize completed activities. This scenario corresponds with the activity recognition feature of the DMN app. Participants were asked to then confirm that the task the smart home recognized was indeed completed. Table 4 shows the frequency of responses by number to question about ease of task completion for confirming an automatically detected smart home task on a Likert scale from 1 (Strongly Satisfied (SS)) to 7 (Strongly Dissatisfied (SD)) from the After Scenario Questionnaire. This task was not included in Iteration 1.

Unlike the activity recognition feature, which was included as a scenario in which participants completed a task, the activity prediction feature was included in Iteration 3 as an open-ended followup question where participant responses were recorded on a digital voice recorder. In this case, participants were given a card showing the predicted task interface and asked to comment on this interface in terms of meaningfulness and usefulness. Unlike the interface shown in Figure 8, the previous iterations of the predicted task interface featured multiple color gradations as the current time approached the predicted time. Two participants commented that some kind of sound accompanying the colors would be helpful as some users may not look at the app frequently enough to catch the color progression as time passed. One participant indicated that the feature would not be helpful for medication specifically as they need to take medication at very specific times of a day. The remaining participant commented that they liked the idea of the color gradation.

In response to a question asking whether the participant would use the app if it was available 


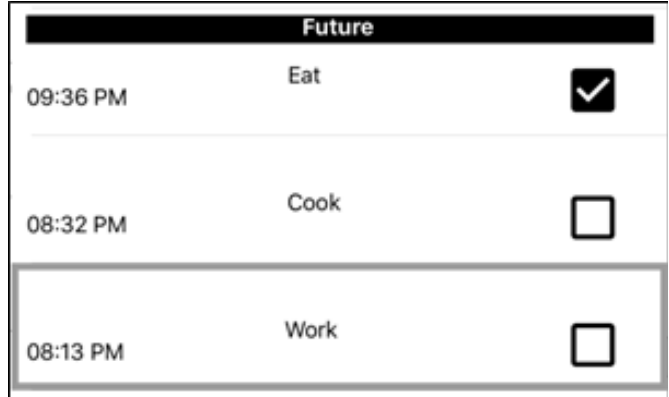

Figure 8. The proposed DMN predicted tasks interface

to the public, $87 \%$ of the participants in Iteration $1,100 \%$ in Iteration 2 , and $100 \%$ in Iteration 3 said they would use the app if it became publicly available.

\section{Smart home pilot test results}

The DMN live smart home features were usability tested in an on-campus smart home test bed called Kyoto (Figure 7), which is located on the Washington State University campus. The two testing sessions included one graduate student participant who was the sole resident of the smart home. The participant tested the smart home features of the DMN for seven days in the first iteration and twenty-one days in the second iteration. The second iteration included updates to the smart home feature interface that were based on participant feedback from the first session. The updated interface is featured in this paper.

The participant was given the DMN app running on a tablet and was asked to incorporate usage of the smart home features into her normal daily routine. For simplicity, the participant was asked to choose three smart home activities of interest from the full set of potential activities (see Table 2 for the set of activities). The activities the participant chose for the first session were Wash Dishes, Enter Home, and Eat. For the second session Eat, Work, and Cook were chosen. During the testing periods, the DMN would automatically notify the participant of recognized activities and also predict when these activities should occur using the interface described in Methods.

At the end of the testing periods, the participant was given a questionnaire based on the PSSUQ to complete with a focus on evaluating the live smart home features. Table 5 shows the participant's responses when asked to evaluate the live smart home based features using a Likert scale from 1 (Not at all satisfied) to 5 (Very satisfied). Participant scores for the live smart home-based features. Satisfaction Score indicates the score obtained using the question "Overall, how satisfied were you with this feature?". Effort Score indicates the score obtained using the question "Overall, how satisfied were you with the amount of effort it took to use this feature?"

\section{Discussion \\ Usability testing discussion}

The DMN app provides a unique approach for utilizing activity recognition and prediction data derived from a smart home to improve traditional interventions used to help older adults with memory impairments remain more functionally independent. We designed the DMN app initially based on a set of criteria established by Díaz-Bossini and Moreno and performed 3 iterations of usability studies ${ }^{26}$. We also partnered the DMN app with activity recognition and prediction data derived from a smart home.

We found that from Iteration 1 to Iteration 3 participants responded more positively to the DMN app in terms of overall usability. This suggests that making modifications based on participant feedback and engaging in an iterative design process helped improve the DMN app in terms of overall usability. It is interesting to note that from Iteration 1 to Iteration 2, participants responded less positively to the DMN in terms of overall usability but from Iteration 2 to Iteration 3 the percent difference in the aggregate score decreased by roughly 25\% (a percent decrease in aggregate score is ideal).

We speculate that this may be due to the fact that older adults without memory impairment were tested in Iteration 1 and a large amount of functionality was added for Iterations 2 and 3 to accommodate participant suggestions. By Iteration 2 the interface had become cluttered. After the feedback from Iteration 2, which was conducted with individuals experiencing memory impairment and caregivers, we simplified the DMN's interface and highlighted only the essential features. The results indicate that focusing on simplification of interfaces when designing for older adults with memory impairment may improve mobile applications in terms of overall usability. This is supported by DíazBossini and Moreno's design criteria, which suggest avoiding unnecessary decoration and color and using simple,

\begin{tabular}{lllll} 
Recognized Activities & 2 & 3 & 3 & 4 \\
Activity Audio Tone & 5 & 4 & 5 & 3 \\
Predicted Activities & 2 & 4 & 3 & 3 \\
\hline
\end{tabular}


straightforward language ${ }^{26}$. It is important to note that the results pertaining to overall usability are very dependent on how usability is characterized in our study (i.e. aggregates of Likert responses to the set of questions drawn from the PSSUQ). There may be other ways to characterize usability that would offer different insights into how an app could be improved.

As Table 4 indicates, in general, participants who completed the smart home activity recognition task were satisfied (1-3's) with this feature in terms of ease of task completion. The participant in Iteration 2 who was strongly dissatisfied with this task (7) indicated that they were not able to easily read the text and the placement was confusing. In Iteration 3 we modified the smart home task's interface based on the participant's feedback. Table 4 shows that for iteration 3, participant's ratings of the smart home task were generally very satisfied $(1-2$ 's) indicating this modification improved the interface. The results indicate that the smart home activity recognition feature's interface was understandable to most participants despite not being in a smart home context. However, the purpose of this feature was initially explained to participants before they completed the scenario. This feature may be useful once users familiarize themselves with it, but its usefulness may not be immediately apparent without some form of explanation.

The participant comments related to activity prediction indicate that introducing a more complex activity aware prompting system could be a valuable addition to activity prediction. In addition to colors that change as the time gets closer to the predicted activity time, some participants indicated that sound throughout the color change would be helpful for those who may not have the app open or within reach at all times. While a sound would get users' attention at some time points, there may be more opportune times to alert users to complete tasks. For example, if the user is in the middle of another activity and hears the sound but is not prompted again at a more convenient moment between activities they may forget the task entirely until they refer to the DMN again. Prompts that are delivered when a person is busy with another activity will likely be ineffective. Furthermore, this feature applied to the 'Take Medication' activity, which may be less useful for older adults who must adhere to a strict medication schedule. However, it may still be helpful for those who follow less strict medication schedules and frequently forget to take their medication.

While the DMN app will need to be developed further for use within a smart home context, by Iteration 3 most participants seemed quite satisfied with the app in terms of overall usability. By Iteration 2 and then continuing into Iteration 3,
$100 \%$ of participants indicated that they would use the app if available. This suggests that with further development the DMN could be used as an effective intervention that acts on smart home data in ways that are meaningful and supportive to older adults with memory impairment.

\section{Smart home pilot test discussion}

For the smart home pilot test, the first iteration results (Table 5) indicated that the smart homebased features of the DMN app needed to be further developed to be effective in a live smart home setting. In the open-ended feedback portion of the questionnaire, the participant indicated concern over the amount of information provided to the user in relation to the DMN app feature interface. The first iteration of the smart home-based features on the DMN app included a large amount of text. The predicted tasks also included a color gradation that would change the border from green to blue, to red as the current time approached the predicted time. The participant indicated that there were too many colors and too much text, making the smart home feature interface difficult to understand.

Comparison of this outcome with the results obtained in the in-lab usability testing sessions supported the need for more in-home usability tests over longer periods of time for intervention technologies. While the participants were generally satisfied with the smart home-based features in a controlled lab setting, the participant who used the live features in a home setting seemed to be confused by the amount of information being provided even when the set of activities was limited. In a lab setting the participants are given small pieces of information in short periods of time. In a smart home setting, the participant is continuously updated with live information as they perform their daily routine, which may become overwhelming.

In order to address these concerns in the second session, we reduced the amount of text, limited the border colors to only red and incorporated more understandable language. We also made the recognized and predicted smart home tasks visually distinctive from each other. For iteration 2, the changes to the interface seem to have improved the predicted and recognized activity interfaces. The participant indicated that these features were more usable. The audio tone feature was rated lower in iteration 2 than in iteration 1. This may be due to the fact that the audio tone feature remained unchanged between sessions.

In addition to Likert style ratings, the participant was also asked open ended questions about the $\mathrm{DMN}$ interface. Following iteration 2, the participant was asked to comment on the perceived accuracy of the predicted and recognized smart 
home activities in terms of timing. The participant was especially impressed with the accuracy of the predicted activities commenting that the algorithm started to "guess what I was going to do before I even began to do it." In addition to a usable interface, it is important that the predicted and detected times for smart home activities are accurate in order to support users in a meaningful way as they complete their daily routine. These preliminary results are encouraging and demonstrate that the AR and prediction algorithms are capable of accurately performing their intended function. To test these features further usability tests with older adult participants in smart home settings will be needed in the future.

\section{Conclusion}

In this paper, we present a novel partnership between smart homes and mobile devices to help older adults manage daily tasks. The digital memory notebook application utilizes a partnership with a smart home infrastructure to make use of real time daily activity recognition and prediction information. Our iterative design process found that the DMN interface and components were generally usable by older adults and that satisfaction with the interface improved based on iterative feedback. We also observe that while iterative design in lab settings is valuable, longer testing periods in actual smart homes are needed to more fully evaluate the usability of the interface. The DMN app is unique because it automatically draws on smart home detected information to monitor and prompt daily activities. The ability of the DMN to interface with smart home technologies will allow older adults to not only interact with a smart home but use its capabilities to improve self-management of critical daily activities.

\section{Acknowledgements}

The authors would like to thank the WSU Cognitive Aging and Dementia Lab and the CASAS Lab. The authors would also like to thank Jenna Beaver, Christine McManus, and Kaylee Spangenberg for helping shape the design of the iOS prototype. Special thanks to Eric Chen for his help with design and development and to Katelyn Brown and Catherine Sumida for their help with administration and design. This work was supported in part by the Office of the Assistant Secretary of Defense for Health Affairs, through the Peer Reviewed Alzheimer's Research Program, Quality of Life Research Award, Funding Opportunity Number: W81XWH-15-PRARP-QUAL under Award No. AZ150096. Opinions, interpretations, conclusions, and recommendations are those of the authors and are not necessarily endorsed by the Department of Defense. This work was also supported in part by the National Institute on Aging under Award No. R25AG046114. This material is also based upon work supported by the National Science Foundation under Grant No. 121407.

\section{References}

1. Humanity's aging. Health and Aging National Institute on Aging [Internet]. 2015; https://www.nia.nih. gov/research/publication/global-health-and-aging/ humanitys-aging; retrieved July 31, 2018

2. Cognitive Impairment: A Call For Action, Now! Center for Disease Control [Internet]. 2016; https:// www.cdc.gov/aging/pdf/cognitive_impairment/ cogimp_poilicy_final.pdf; retrieved July 31, 2018

3. Gauthier S, Reisberg B, Zaudig $M$, Petersen RC, Ritchie K, Broich K, Belleville S, Brodaty H, Bennett D, Chertkow H, Cummings JL, de Leon $M$, Feldman $H$, Ganguli $M$, Hampel $H$, Scheltens $\mathrm{P}$, Tierney MC, Whitehouse P, Winblad B. Mild cognitive impairment. The Lancet [Internet]. 2006 Apr [cited 2018 Apr 5];367(9518):126270. http://linkinghub.elsevier.com/retrieve/pii/ S0140673606685425; retrieved July 31, 2018

4. 2016 Alzheimer's disease facts and figures. Alzheimers Dement [Internet]. 2016 Apr [cited 2017 Sep
6];12:459-509. http://linkinghub.elsevier.com/retrieve/ pii/S1552526016000856; retrieved July 31, 2018

5. Farias ST, Mungas D, Reed BR, Harvey D, CahnWeiner D, DeCarli C. MCl is Associated With Deficits in Everyday Functioning: Alzheimer Dis Assoc Disord [Internet]. 2006 Oct [cited 2018 Apr 5];20(4):217-23. https://insights.ovid.com/crossref ?an=00002093-200610000-00008; retrieved July 31,2018

6. Schmitter-Edgecombe M, Howard JT, Pavawalla SP, Howell L, Rueda A. Multidyad Memory Notebook Intervention for Very Mild Dementia: A Pilot Study. Am J Alzheimers Dis Dementiasr [Internet]. 2008 Oct [cited 2017 Sep 6];23(5):477-487. http://journals.sagepub.com/doi/10.1177/1533317508320794; retrieved July 31, 2018

7. Greenaway MC, Duncan NL, Smith GE. The memory support system for mild cognitive impairment: randomized trial of a cognitive rehabilitation intervention: $\mathrm{MCl}$ rehabilitation. Int J Geriatr Psychiatry [Internet]. 2013 Apr [cited 2018 Apr 5];28(4):402409. http://doi.wiley.com/10.1002/gps.3838

8. Chen L, Nugent CD, Wang H. A Knowledge-Driven Approach to Activity Recognition in Smart Homes. IEEE Trans Knowl Data Eng [Internet]. 2012 Jun [cited 2018 Apr 5];24(6):961-974. http://ieeexplore.ieee. org/document/5710936/; retrieved July 31, 2018

9. Wan J, O'Grady MJ, O'Hare GMP. Dynamic sensor event segmentation for real-time activity recognition in a smart home context. Pers Ubiquitous Comput [Internet]. 2015 Feb [cited $2018 \mathrm{Apr}$ 5];19(2):287-301. http://link.springer.com/10.1007/ s00779-014-0824-x; retrieved July 31, 2018

10. Cook DJ, Das SK. How smart are our environments? An updated look at the state of the art. Pervasive Mob Comput [Internet]. 2007 Mar [cited 2018 Apr 9];3(2):53-73. http://linkinghub.elsevier.com/retrieve/ pii/S1574119206000642; retrieved July 31, 2018

11. Wilson C, Hargreaves T, Hauxwell-Baldwin R. Smart homes and their users: a systematic analysis and key challenges. Pers Ubiquitous Comput [Internet]. 2015 Feb [cited 2018 Apr 5];19(2):463-476. 
http://link.springer.com/10.1007/s00779-0140813-0; retrieved July 31, 2018

12. Rafferty J, Nugent CD, Liu J, Chen L. From Activity Recognition to Intention Recognition for Assisted Living Within Smart Homes. IEEE Trans HumMach Syst [Internet]. 2017 Jun [cited 2018 Apr 11];47(3):368-379. http://ieeexplore.ieee.org/document/7807210/; retrieved July 31, 2018

13. Gayathri KS, Easwarakumar KS, Elias S. Probabilistic ontology based activity recognition in smart homes using Markov Logic Network. Knowl-Based Syst [Internet]. 2017 Apr [cited 2018 Apr 11];121:173184. http://linkinghub.elsevier.com/retrieve/pii/ S0950705117300370; retrieved July 31, 2018

14. Baruah RD, Singh M, Baruah D, Misra IS. Predicting activity occurrence time in smart homes with evolving fuzzy models. In: 2017 IEEE International Conference on Fuzzy Systems (FUZZ-IEEE). 2017. p. 1-5.

15. Schmitter-Edgecombe M, Fahy JF, Whelan JP, Long CJ. Memory remediation after severe closed head injury: Notebook training versus supportive therapy. J Consult Clin Psychol [Internet]. 1995 [cited 2017 Sep 6];63(3):484-489. http://doi.apa.org/ getdoi.cfm?doi=10.1037/0022-006X.63.3.484; retrieved July 31, 2018

16. Cicerone KD, Langenbahn DM, Braden $C$, Malec JF, Kalmar K, Fraas M, Felicetti T, Laatsch L, Harley JP, Bergquist T, Azulay J, Cantor J, Ashman T. Evidence-Based Cognitive Rehabilitation: Updated Review of the Literature From 2003 Through 2008. Arch Phys Med Rehabil [Internet]. 2011 Apr [cited 2018 Apr 11];92(4):519-530. http://linkinghub.elsevier.com/retrieve/pii/S0003999310009500; retrieved July 31, 2018

17. Schmitter-Edgecombe M, Dyck DG. Cognitive Rehabilitation Multi-family Group Intervention for Individuals with Mild Cognitive Impairment and Their Care-Partners. J Int Neuropsychol Soc [Internet]. 2014 Oct [cited 2017 Sep 6];20(09):897908. http://www.journals.cambridge.org/abstract_ S1355617714000782; retrieved July 31, 2018

18. Vemuri S, Schmandt C, Bender W, Tellex S, Lassey B. An Audio-Based Personal Memory Aid. In: Davies N, Mynatt ED, Siio I, editors. UbiComp 2004: Ubiquitous Computing [Internet]. Berlin, Heidelberg: Springer Berlin Heidelberg; 2004 [cited 2017 Sep 6]. p. 400-17. http://link.springer.com/10.1007/9783-540-30119-6_24; retrieved July 31, 2018

19. Tan D, Berry E, Czerwinski M, Bell G, Wood K. Save everything: supporting human memory with a personal digital lifetime store. Pers Inf Manag. 2007;90

20. Czerwinski M, Gage DW, Gemmell J, Marshall CC, Pérez-Quiñones MA, Skeels MM, Catarci T. Digital memories in an era of ubiquitous computing and abundant storage. Commun ACM [Internet]. 2006 Jan 1 [cited 2017 Sep 6];49(1):44. http://portal.acm. org/citation.cfm?doid=1107458.1107489; retrieved July 31, 2018

21. M Tsui K, A Yanco H. Prompting Devices: A Survey of Memory Aids for Task Sequencing. QoLT Int

\section{Symp Intell Syst Better Living. 2010;}

22. Kapur N, Glisky EL, Wilson BA. Technological memory aids for people with memory deficits. Neuropsychol Rehabil [Internet]. 2004 Mar [cited 2017 Sep 6];14(1-2):41-60. http://www.tandfonline.com/doi/abs/10.1080/09602010343000138; retrieved July 31, 2018

23. Feuz KD, Cook DJ, Rosasco C, Robertson K, Schmitter-Edgecombe M. Automated Detection of Activity Transitions for Prompting. IEEE Trans Hum-Mach Syst [Internet]. 2015 Oct [cited 2017 Sep 6];45(5):575-585. http://ieeexplore.ieee.org/ document/6949090/; retrieved July 31, 2018

24. Okoshi T, Ramos J, Nozaki H, Nakazawa J, Dey AK, Tokuda H. Attelia: Reducing user's cognitive load due to interruptive notifications on smart phones. In: 2015 IEEE International Conference on Pervasive Computing and Communications (PerCom). 2015. p. 96-104

25. Ho J, Intille SS. Using Context-aware Computing to Reduce the Perceived Burden of Interruptions from Mobile Devices. In: Proceedings of the SIGCHI Conference on Human Factors in Computing Systems [Internet]. New York, NY, USA: ACM; 2005. p. 909-918. (CHI '05). http://doi.acm. org/10.1145/1054972.1055100; retrieved July 31, 2018

26. Díaz-Bossini J-M, Moreno L. Accessibility to Mobile Interfaces for Older People. Procedia Comput Sci [Internet]. 2014 [cited 2017 Sep 4];27:57-66. http://linkinghub.elsevier.com/retrieve/pii/S1877050914000106; retrieved July 31, 2018

27. Cook D, Krishnan NC. Activity learning: Discovering, recognizing, and predicting human behavior from sensor data. 2015. pp. 1-257.

28. Krishnan NC, Cook DJ. Activity recognition on streaming sensor data. Pervasive Mob Comput [Internet]. 2014 Feb [cited 2018 Apr 5];10:138154. http://linkinghub.elsevier.com/retrieve/pii/ S1574119212000776; retrieved July 31, 2018

29. Minor BD, Doppa JR, Cook DJ. Learning ACtivity Predictors from Sensor Data: Algorithms, Evaluation, and Applications. IEEE Trans Knowl Data Eng [Internet]. 2017 Dec 1 [cited 2018 May 11];29(12):2744-2757. http://ieeexplore.ieee.org/ document/8031060/; retrieved July 31, 2018

30. Minor B, Doppa JR, Cook DJ. Data-Driven Activity Prediction: Algorithms, Evaluation Methodology, and Applications. In: Proceedings of the 21th ACM SIGKDD International Conference on Knowledge Discovery and Data Mining [Internet]. New York, NY, USA: ACM; 2015. p. 805-814. (KDD '15). http://doi.acm.org/10.1145/2783258.2783408; retrieved July 31, 2018

31. Lewis JR. Psychometric Evaluation of the Post-Study System Usability Questionnaire: The PSSUQ. Proc Hum Factors Ergon Soc Annu Meet [Internet]. 1992 Oct [cited 2018 Apr 5];36(16):1259-1260. http://journals.sagepub.com/doi/10.1177/154193129203601617; retrieved July 31, 2018 